\title{
Algunos aspectos de las contribuciones especiales en la Ley del Suelo
}

\author{
por \\ FEDERICO ROMERO HERNANDEZ \\ Secretario de $x^{2}$ categoría de Administración local
}

SUMARIO: I. Introducción.-U. Las contribuciones especiales. A) Idea de su concepto y fundamento. B) Clases de contribuciones especiales en la Ley de Régimen local y en la del Suelo. C) Dificultades de aplicación en la práctica.-III. Las contribuciones especiales dentro de los sistemas de actuación que se establecen en la Ley del Suelo. A) Los tres primeros sistemas de ejecución y las contribuciones especiales. B) El sistema de cesión de terrenos viales con imposición de contribuciones especiales. Especialidades que se observan en la regulación de contribuciones especiales por la Ley del Suelo.-IV. Conclusión.

\section{INTRODUCCION}

En muchos de los trabajos que sobre la Ley del Suelo se han realizado se ha puesto de manifiesto la dificultad que su estudio y aplicación adecuada entraña. Aparte su complejidad y extensión, es el haber surgido casi de la nada el primero de sus inconvenientes. Como ha puesto de manifiesto García dE ENTERría (1), «es tal su originalidad en nuestro Derecho, sus regulaciones introducen novedades de tanto bulto en la situación anterior, que ha de ser preciso un poderoso esfuerzo de adaptación de las conciencias por parte de propietarios, administrativos, funcionarios, arquitectos, juristas y jueces, para que puedan extraerse de tan

(1) «La Ley del Suelo y el futuro del urbanismo», en Problemas actuales de Régimen local. Publicaciones del Instituto García Oviedo, Sevilla, pág. 96. 
importante cuerpo legal todas las posibilidades que indudablemente reposan en él». Como es lógico, el aspecto económico y hacendístico no escapa a estas innovaciones. El presente trabajo se refiere precisamente a un sector de estos aspectos: el referente a las contribuciones especiales.

Importa, sin embargo, poner de manifiesto desde ahora que la originalidad en este respecto no está tanto en estrictas modificaciones o peculiaridades de la contribución especial en sí, cuanto en conseguir los fines que fundamentan y justifican a éstas por otras vias que al ser, en definitiva, más directas, conceden una mayor agilidad y proporcionan un mayor realismo en la consecución de medios económicos. $\mathrm{Y}$ si, como es sabido, han sido precisamente las dificultades económicas las que han demorado la realización de las urbanizaciones, el asunto aquí tratado adquiere una importancia práctica de primer orden.

\section{LAS CONTRIBUCIONES ESPECIALES}

\section{A) Idea de su concepto y fundamento.}

Empezamos por dar varios conceptos doctrinales, tanto de autores españoles como extranjeros, para deducir inmediatamente la justificación y fundamento técnico de la contribución especial, ya que entendemos que es esencial destacarlos a efectos de iluminar ampliamente la consideración que en la Ley del Suelo tiene.

NAHARRo (2) nos dice que es «el pago obligatorio del coste de la parte divisible de un servicio público preponderantemente indivisible, que beneficia especialmente a los bienes inmuebles de una zona, y cuyo importe se satisface de una vez para siempre, aunque pueda ser fraccionado». Más claras, a los efectos que a nosotros nos interesa, son las definiciones que AlBI cita en su Derecho municipal comparado del mundo hispánico (3), a saber, las de:

a) EINAudi (4), que nos dice que «es la compensación pagada

(2) Lecciones de Hacienda pública, pág. 76.

(3) Pág. 512.

(4) Principios de Hacienda pública, pág. 78. 
con carácter obligatorio al ente público con ocasión de una obra realizada por él con fines de utilidad pública, pero que proporciona también ventajas especiales a los particulares propietarios de los bienes inmuebles».

b) Seligmaiv (5), en el mismo sentido, considera que «es una contribución forzosa establecida de modo proporcional a los beneficios especiales obtenidos, para cubrir los gastos de una mejora concreta de la propiedad, llevada a cabo en interés público».

De estas dos definiciones deduce AlBI (6) que, así como en el precio en la tasa el beneficio es exclusivo para los usuarios y perfectamente divisible entre los mismos, y en el impuesto es básico el interés general no individualizable, en la contribución encontramos un planteamiento mixto, en que juegan ambos factores. Ocurre a veces que en determinado sector de obras públicas la utilidad se presenta dividida: por una parte, tenemos el beneficio genérico que redunda sobre la colectividad y que no es fraccionable, por lo cual ha de ser pagado mediante exacciones que soporte, de modo general, la propia colectividad; pero, por otra parte, existe un beneficio que recae de forma directa sobre cierta categoría de personas o, mejor dicho, de propietarios de inmuebles situados en una determinada zona territorial, los cuales han de retribuir este provecho mediante contribuciones especiales. En un sentido análogo se pronuncia muy claramente G. DEL VALLE YANGUAS (7) : «Si un Ayuntamiento ejecuta obras, realiza instalaciones o implanta o amplía un servicio que, a más del interés general que reporte para la colectividad sometida a su gobierno y administración, proporciona un mayor grado de utilidad, utilidad diferencial que se traduce, bien en un aumento de valor de los inmuebles sitos en el área de influencia de aquellas obras, instalaciones o servicios, ya en un beneficio especial a personas o clases determinadas, aparece plenamente justificado el derecho de establecer una exacción destinada, cuando más, a cubrir el coste de ejecución de las mismas y exigible de los propietarios de los inmuebles y de las personas que lograron la ventaja especial».

(5) Essays in Taxation, New York and London, 1895.

(6) Ob. y pág. cits.

(7) Derechos y tasas, contribuciones especiales. 
En definitiva, con la contribución especial se rescata, mediante un pago que se hace de una vez para siempre (aunque este pago único puede fraccionarse), el coste de una obra pública que produce un aumento de valor de ciertas fincas o, simplemente, beneficia a personas o clases determinadas. Como veremos más adelante, este fundamento básico de la contribución especial, que tiende a repartir equitativamente las cargas públicas y que evita el enriquecimiento indebido de ciertos propietarios que se benefician especialmente de obras que, por ser públicas, deben extender sus beneficios a toda la comunidad, constituye su principio esencial en la Ley del Suelo, en el sentido de que calificándose el urbanismo como una función pública exclusiva (8), los aumentos de valor en las fincas que se producen como consecuencia de la urbanización se encauzan siempre para conseguir, ante todo, un beneficio de la comunidad por los distintos caminos que se establecen.

B) Clases de contribuciones especiales en la Ley de Régimen local y en la del Suelo.

Como es sabido, el artículo 451 de la Ley de Régimen local distingue por su procedencia las contribuciones especiales que se determinan por razón de una plus valía, y la contribución especial que, independientemente de este aumento de valor, procede en razón a un beneficio de personas o clases determinadas, o que se provocan de un modo especial por las mismas.

No es objeto de este trabajo el estudio de las contribuciones especiales en general ni, por tanto, debemos detenernos en la regulación que la Ley de Régimen local hace de estas contribuciones. Este Derecho positivo vigente lo damos por supuesto. Nos interesa aquí destacar ahora las consecuencias que se deducen de la comparación de este artículo 451 de la Ley de Régimen local con el artículo 183, párrafo 1.o, de la Ley del Suelo, ya que nos servirán para hacer una primera precisión del grupo de contribuciones especiales a que se refiere esta Ley de 12 de mayo

(8) Sobre este punto, Garcia de Enterría, ob. cit., pág. 128. 
de 1956, para más adelante hacer un detenido estudio de las especialidades que se encuentran en su conjunto normativo.

El párrafo 1. del artículo 183 de la Ley del Suelo dice: «Cuando las obras, instalaciones o servicios ejecutados por el Ayuntamiento beneficiasen especialmente a personas o clases determinadas, o se provocasen de un modo especial por las mismas, aunque no existieran aumentos determinados de valor, la imposición por contribuciones especiales será, como máximo, del 90 por 100 del coste total, con carácter uniforme».

Efectivamente, este párrafo 1. del artículo 183 de la Ley del Suelo recoge el grupo de contribuciones especiales a que se refiere el apartado b) del párrafo 1. del artículo 451, a lo que añade la fijación del tope máximo de la cuota aplicable con carácter uniforme. El citado apartado se reproduce taxativamente.

Por otra parte, salta a la vista que la consideración por separado del apartado b) del párrafo 1. del artículo 451, su formulación independiente (que es lo que hace el artículo 183 de la Ley del Suelo), lleva consigo el tener en cuenta no sólo las contribuciones por un beneficio especial de determinadas personas o que se provocan por las mismas («Demás contribuciones especiales», en la L. R. L.), sino las «contribuciones de plus valía», ya que a fortiori se deduce de la expresión «aunque no existieran aumentos determinados de valor». Ello es una consecuencia de lógica jurídica que se deduce del hecho de que el apartado b) del artículo 451 de la L. R. L. contiene en sus términos una ampliación del apartado $a$ ) dando por supuesto éste y siendo de ámbito más extenso.

Naturalmente, concluimos que en la Ley del Suelo se tienen, por tanto, en consideración las contribuciones especiales, tanto de plus valía como las demás a que se refiere la L. R. L. en el apartado b) del 451. Además, la cuota que se establece con carácter uniforme es precisamente la que se establece en plus valía en la L. R. L.

C) Dificultades de aplicación en la práctica.

Inspirándose en la Ley prusiana de contribución comunal, se 
ha dicho por algunos autores (9) que las contribuciones especiales han sido el refuerzo más sólido de las Haciendas de nuestras ciudades y que por estar cargados de justicia mereció general asentimiento. «Fueron muchos los millones-nos dice C. MartíN RETortmlo (10) - que por efectos de la acción de los Ayuntamientos engrosaron los patrimonios particulares de los propietarios del suelo de nuestras ciudades».

$Y$, en definitiva, las contribuciones especiales venían, con su incorporación a los medios de recaudación de las Corporaciones locales, a evitar que este enriquecimiento, polarizado en un sector, se sufragaran a tipos de gran dureza, fundamentalmente por una clase media conocedora de esa elevación, injustificada en muchos casos, de los capitales ajenos.

Sin embargo, pese a la brillantez de este refuerzo y a la justicia de su incorporación-como ha dicho Rodríguez Moro-, las contribuciones especiales «no han rendido en el orden práctico el fruto que cabía esperar de un tan bien tratado cuerpo legal como el establecido al efecto» (11). El autor citado pone de manifiesto lo dificultoso del expediente de imposición de las contribuciones especiales en cuanto a múltiples de sus actuaciones: fijación de superficies afectadas, aumentos de valor..., etc., o los recovecos desde donde puede fácilmente atacársele para dejar sin efecto la imposición o disminuir la cuota en tal porcentaje que resulte ridícula la proporción al elevado costo de la obra.

Por otra parte, la reclamación y los recursos contra la aplicación de contribuciones especiales constituyen también serios inconvenientes a su efectividad práctica, en cuanto que en los referentes a los últimos-como se ocupa de resaltar el mismo RoDRÍGUEZ MORO (12)--, pocas veces el fallo ha sido favorable para

(9) Por ejemplo, C. MARTín-Retortillo, Comentario a la Ley de Régimen local, Aguilar, 1958, pág. 314.

(10) Ob. y pág. cits.

(11) N. RODRíguez MORo: «Las contribuciones especiales y los demás medios que otorga la Ley del Suelo para la ejecución de los planes y proyectos urbanísticos», R.E.V.L., 106, pág. 501.

(12) Ob. y pág. cits. 
la Corporación municipal, aunque recientemente se haya producido alguno, como el de 15 de octubre de 1960.

En cuanto a las reciamaciones ante los Tribunales Económicoadminisirativos provinciales, ha de tenerse en cuenta también por el hecho de la suspensión y consiguiente aplazamiento de pago de las liquidaciones impugnadas hasta que sea resuelta la reclamación contra la aplicación de la contribución especial (por virtud de lo dispuesto en el artículo 727 de la L. R. L., como ha señalado recientemente una sentencia de 27 de abril de 1961), determinan nuevas dilaciones a la efectividad de la contribución.

En definitiva, se puede afirmar, por tanto, que a pesar del celo desplegado por algunos funcionarios y la experiencia obtenida por los Ayuntamientos, que Rodríguez Moro señala (13), las dificultades expuestas hacen que en la práctica la contribución especial no haya rendido todos los frutos que de ella se esperaban.

\section{LAS CONTRIBUCIONES ESPECIALES DENTRO DE LOS SISTEMAS DE ACTUACION QUE SE ESTABLECEN EN LA LEY DEL SUELO}

Como hemos tenido ya ocasión de apuntar, la mayoría de los autores que se han referido en sus estudios a la Ley del Suelo señalan el avance considerable que esta Ley, con sus sistemas de ejecución, representa al compararse con el sistema de contribuciones especiales. En definitiva, esto no es más que una deducción del principio fundamental de esta Ley: convertir la urbanización en una facultad de carácter público. Y ello incide en la esencia misma del fundamento de la contribución especial. En la Ley se establecen una serie de valores: inicial o rústico, expectante, urbanístico y comercial, que constituyen una escala cuyos beneficios se afectan al costeamiento de obras de interés público, ăistribuyéndose así entre la comunidad y evitando que lo sea exclusivarnente de un grupo determinadc. Como ya hemos puesto antes de manifiesto, ss esto lo que, no muy eficazmente, trataba de ccrseguir la contribución especial.

(13) Cb. y pág. cits. 
Ahora bien, de los cuatro sistemas de ejecución que se arbitran: a), sistema de cooperación; $b$ ), de expropiación total de los terrenos; $c$ ), de compensación, y $d$ ), de cesión de terrenos viales, en el último se sigue utilizando la contribución especial. Conviene, por tanto, continuar nuestro estudio de esta exacción en la Ley del Suelo partiendo de la siguiente distinción:

A) El avance de los tres primeros sistemas de ejecución en relación con la contribución especial.

B) El sistema de cesión de terrenos viales con imposición de contribuciones especiales.

A) Los tres primeros sistemas de ejecución y las contribuciones especiales.

1) Aparte de las dificultades prácticas del cobro de la contribución especial a que ya nos hemos referido, con los sistemas de ejecución que estudiamos, como destaca GARCía DE ENTERRÍ (14), «estamos ante una figura que, no obstante su aparente extrañeza, responde a los puros principios de la contribución especial, de la que se ha suprimido con fines de eficacia el iter inutilis que era: expropiación con indemnización seguida de resarcimiento de esta indemnización por contribución dineraria impuesta a los propietarios del sector».

Este autor parece que concreta este «ahorro de camino inútil» en la cesión de los terrenos precisos para las vías urbanas y zonas verdes. Entendemos que los atajos se producen, aunque lógicamente de distinta manera, en cada uno de los sistemas de ejecución y siempre respondiendo a los puros principios de la contribución especial:

a) En el sistema de cooperación existe una acentuada actuación mixta. El ahorro del camino se verifica no sólo en cuanto que se cede la zona vial y verde, sino porque se contribuye a los gastos de su urbanización.

b) En el sistema de compensación existe una acusada intervención del lado de los particulares. Si se nos permite, podríamos decir que se constituye una sociedad incidental con una masa

(14) Ob. cit., págs. 119 y 120. 
común de terrenos para viales y zonas verdes que implica una realización total por parte de los propietarios de dicho terreno. El atajo no puede ser más corto.

c) En el sistema de expropiación la actuación se vuelca del lado de la Administración (15). En realidad, aquí el camino es totalmente distinto, ya que se hace tabla rasa con el terreno, al expropiarlo todo, tanto la zona vial y verde como los edificables, con los aprovechamientos que contengan.

Pero lo que sí es evidente es que, de todas formas, el resultado es el mismo que pretendía lograr la contribución especial: el $\tan$ repetido reparto del beneficio que el urbanismo reporta entre la comunidad.

2) Se consigue, pues, el mismo fin que perseguia una exacción por un camino distinto y más corto. ¿Qué calificación jurídico-tributaria cabe hacer de la técnica que se utiliza para conseguir esta finalidad? En principio es necesaria de nuevo la discriminación del problema según los distintos sistemas de ejecución. Creemos que es evidente que en el caso del sistema de expropiación no cabe plantear esta cuestión en absoluto. En otras palabras, si queremos estudiar las consecuencias que en orden a la calificación jurídico-tributaria se deducen del hecho de que, realizándose unas obras de urbanización, se produzca un beneficio o un aumento de valor en unas determinadas fincas y no se proceda al cobro de las contribuciones especiales en dinero a causa de la colaboración o cooperación de los particulares afectados, es lógico que en el caso del sistema de expropiación no nos hagamos esta pregunta, ya que falta el supuesto que le sirve de base.

Concretamos, pues, la cuestión ahora a los sistemas de cooperación y de compensación.

a) En principio creemos que cabe descartar la idea de una exención tributaria de contribuciones especiales a favor de los propietarios, como premio a la cesión de los terrenos y al costeamiento o contribución en el pago de las urbanizaciones. $\mathrm{Y}$ ello

(15) Aunque la Ley, en el artículo 121, párrafo 1.?, considera que pueden ser también expropiantes las gersonas privadas a quienes autorice la Comisión Central de Urbanismo, creemos que esto se dará con poca frecuencia en la práctica. 
porque: en primer lugar-y como dice César ArBIÑana (16)-, «tanto en las tasas como en las contribuciones especiales no caben las exenciones en su exacto significado, porque las primeras son siempre voluntarias y son contraprestaciones a una actividad administrativa, y las segundas constituyen, al fin, el rescate del coste parcial de una obra pública que mejora cosa cuya titularidad dominical ostenta el sujeto obligado al pago de la contribución especial». Esto hay que coordinarlo, sin embargo, con lo que el mismo autor dice en otro lugar (17) al tratar de los exceptuados de las contribuciones especiales previstos en los artículos 468 y 472 de la L. R. L. Pero, sobre todo, lo que importa tener en cuenta es que si la exención tributaria (18) existe "cuando en una «situación-base» de imposición se dan, además de los hechos imponibles, presupuestos integrantes de aquélla, uno o más requisitos exigidos por la Ley para el disfrute de un régimen de excepción en cuanto a la prestación tributaria correspondiente», es evidente que no estamos en el caso que nos planteamos. Tampoco estamos en el caso de inmunidad o de remisión impositiva.

b) Más cerca parece que estamos de un caso de compensación. En el artículo 454 de la L. R. L. se dice en su párrafo 3. que si la ejecución de las obras, instalaciones o servicios fueren auxiliados por subvenciones $\mathrm{u}$ otras cooperaciones del Estado, de la Provincia o de otra corporación o de particulares, el importe de estos recursos se descontará del coste de las obras, salvo lo dispuesto en el artículo siguiente. Pues bien, en el artículo 455 de esta misma Ley se dice que si los auxilios a que se refiere el párrafo del artículo que acabamos de citar se otorgasen por quien tuviere la obligación de contribuir especialmente, el importe de tales auxilios no será deducido del coste total de las obras, instalaciones o servicios, a los efectos de la determinación de la suma de las contribuciones exigibles, sino que en cada caso será objeto de especial compensación en el importe de la cuota de la

(16) Voz «Exención del impuesto», tomo IY de la N. Enciclopedia Juridica Seix, págs. 687 y sigs.

(17) Voz «Contribución especial», tomo V de la N. E. J. S., págs. 687 $\mathrm{y}$ siguientes.

(18) Voz «Exención del impuesto»; Albinana, cit. 
respectiva persona o entidad (párrafo 1.). Este caso de compensación se entiende sin perjuicio del derecho a la renuncia, antes del señalamiento de ia cuota, por parte del interesado (párrafo 5.9 del mismo artículo 455 ).

Sin embargo-como señala Albiñana al comentar estos textos legales (19) -, el supuesto que aquí contemplamos no es jurídicamente una compensación sino en cuanto asi lo dispone la Ley, aunque en su entraña económica el auxilio puede ser considerado como verdadero anticipo de la cuota contributiva especial, que ha de ser fijada a la entidad o particular que subvencionó o cooperó.

c) García de Enterría (20) considera que las dos innovaciones fundamentales en orden a la contribución especial está precisamente en convertirse en contribuciones: $a$ ), ex ante; $b$ ), en especie. Pues bien, a la vista de lo que acabamos de decir al referirnos hace un momento a la posible compensación y que según ALBIÑana podría ser más bien un anticipo de la cuota contributiva, podriamos pensar que, efectivamente, esto se coordinaba con la contribución anticipada y en especie por la cesión de terrenos viales y verdes que el citado profesor GARCía DE ENTERRÍA señala. Ahora bien, si nos fijamos en la regulación legal en relación con el problema que nos planteamos, deducimos que si comparamos el sistema de cooperación con el llamado sistema de cesión de terrenos viales, en ambos existe esa cesión de terrenos para viales y zonas verdes y, sin embargo, sólo en el primero de los comparados no se impone la contribución especial. Parece que puede deducirse aqui, por tanto, que más que contribuir en especie, en el caso de sistema de cooperación estamos en un caso de anticipo de la cuota contributiva especial por el dinero aportado para costear las obras de urbanización (artículo 115, en relación con el 117, de la Ley del Suelo).

El sistema de compensación aparece fundamentalmente diferenciado del de ccoperación en la Ley por la actividad total aue los perriculares desarrollan en la urbanización al formar ia masa común en aquel sistema. Sin ernbango, en cuanto al probiema que

(19) Voz «Contribuc:ón especial» cit.

(20) Ob. cit., págs. 118 y sigs. 
nosotros nos estamos planteando, no añade nada a lo que hemos dicho sobre el sistema de cooperación, ya que al sobrepasar a éste en cuanto a los medios aportados por los particulares, engloba la calificación que de este sistema hemos hecho.

B) El sistema de cesión de terrenos viales con imposición de contribuciones especiales. Especialidades que se observan en la regulación de contribuciones especiales por la Ley del Suelo.

a) Los criterios de prioridad que se establecen en la Ley.El párrafo 2. del artículo 113 de la Ley del Suelo deja libertad al Ayuntamiento u órgano gestor para elegir el sistema de ejecución que más convenga. Sin embargo, y con carácter subsidiario, si no precisa el sistema a seguirse en el polígono el plan de ordenación, o no lo determina el órgano gestor de la urbanización, declara preferentemente el de cesión de terrenos viales con imposición de contribución especial, cuando se trate de sectores parcialmente urbanizados y edificados. Es evidente que, al menos en general, este sistema es el más adecuado cuando en la base territorial a urbanizar existen realizaciones parciales.

b) Vamos a hacer un análisis de las particularidades que la contribución especial tiene en la Ley del Suelo con respecto a la regulación general de la $L$. $R$. $L$. En todo caso debemos tener siempre presentes las consideraciones que en torno a las dos clases de contribuciones especiales hicimos al principio.

$\left.a^{\prime}\right)$ En primer lugar, ya hemos señalado antes cómo se establece con carácter uniforme una cuota máxima del 90 por 100 del coste de la obra, instalación o servicio (artículo 183, párrafo 1.?, de la Ley del Suelo). El 10 por 100 que resta es, lógicamente, la participación en el beneficio o el aumento de valor que el propietario gravado tiene. Consecuente con esta disposición, el párrafo 2. del mismo artículo 183 puntualiza que no serán de aplicación las limitaciones establecidas en el artículo 470 de la L. R. L. Como es sabido, este artículo establece una especificación de cuotas máximas para las contribuciones especiales que no se establezcan por aumentos determinados de valor.

$\left.b^{\prime}\right)$ En el artículo 130 de la Ley del Suelo se establece la im- 
portante especialidad a que nos referíamos antes, citando a GARCÍA DE ENTERRÍA: la contribución especial ex ante. Acordada la ejecución de las obras, el Ayuntamiento podrá exigir con seis meses de anticipación el pago de las contribuciones especiales que hubieran de devengarse, en proporción a los gastos que para el mismo período se prevean. Constituye uno de los muchos intentos que en la Ley del Suelo se hacen para paliar los defectos que en orden al realismo económico tenían antes las contribuciones especiales.

c) El párrafo 3. del artículo 130 establece que el Ayuntamiento no podrá dejar de imponer y percibir las contribuciones especiales en ningún caso. RODRíGUez Moro (21) observa que parece que al exigir que, en todo caso, se impongan las contribuciones especiales se va más allá de lo que exige la L. R. L., la cual, en su artículo 451, solamente establece con carácter obligatorio la imposición y la exacción de las contribuciones especiales cuando haya aumentos determinados de valor. Efectivamente, pese a que, como pusimos de manifiesto antes, tanto las contribuciones especiales de plus valía como las determinadas por beneficio especial tienen acogida en la Ley del Suelo, la situación que dentro de su texto tiene el precepto que analizamos hace suponer que en el caso de urbanizaciones por el sistema de cesión de terrenos viales, la contribución especial se impondrá obligatoriamente, haya aumento determinado de valor o sólo se produzca su beneficio especial a personas o clases determinadas.

\section{CONCLUSION}

A través de todas las consideraciones que hasta aquí hemos realizado hemos intentado resaltar cómo, convertido el urbanismo en una función exclusivamente pública, se llega por diversos caminos a una finalidad que justificó y justifica a la contribución especial: evitar el beneficio especial exclusivo de determinadas personas a causa de obras que por ser de carácter público deben extender sus beneficios a toda la comunidad. Esta finalidad, ner-

(21) Ob. cit., pág. 520. 
vio importantísimo en la Ley del Suelo y base que fundamenta la contribución especial a la vez, se consigue mediante una técnica que tiene una calificación y unos efectos jurídico-tributarios:

a) En los sistemas de ejecución que no comportan contribución especial se consigue por diversos caminos, más directos que la propia contribución especial, con una mayor agilidad económica y que, desde el punto de vista de su calificación jurídicotributaria, creemos que dan lugar a un caso de anticipo de la cuota contributiva especial mediante el dinero que se aporta para costear las obras de urbanización. En el caso de sistema de expropiación, entendemos que falta el supuesto-base para esta calificación.

b) Por otra parte, aun en el caso de imposición de contribución especial en el sistema de cesión de terrenos para viales, la Ley del Suelo, con mayor realismo económico que en sistemas anteriores, refuerza a la propia contribución especial con el cobro anticipado y la cesión obligatoria de los terrenos para viales.

Como en otros muchos aspectos de la Ley del Suelo, esperamos que las indudables posibilidades que lleva implícitas encuentren su efectividad en la aplicación. 